\title{
The New Era of Biologics in Atopic Dermatitis: A Review
}

\author{
Simon Schneider ${ }^{1}$, Linda Li ${ }^{1}$, Alexander Zink ${ }^{1,2}$
}

1 Technical University of Munich, School of Medicine, Department of Dermatology and Allergy, Munich, Germany

2 Division of Dermatology and Venereology, Department of Medicine Solna, Karolinska Institute, Stockholm, Sweden

Key words: atopic dermatitis, therapy, biological, dupilumab

Citation: Schneider S, Li L, Zink A. The new era of biologics in atopic dermatitis: a review. Dermatol Pract Concept. 2021;11(4):e2021144. DOI: https://doi.org/10.5826/dpc.1104a144

Accepted: October 19, 2021; Published: October, 2021

Copyright: $\odot 2021$ Schneider et al. This is an open-access article distributed under the terms of the Creative Commons Attribution License BY-NC-4.0, which permits unrestricted noncommercial use, distribution, and reproduction in any medium, provided the original authors and source are credited.

Funding: None.

Competing interests: None.

Authorship: SS and LL have equally contributed to this publication.

Corresponding author: Simon Schneider, Technical University of Munich, School of Medicine Department of Dermatology and Allergy, Munich, Germany. Email: simon.a.schneider@tum.de

This article is part of a series of reviews dedicated to Atopic Dermatitis, guest edited by Prof Anna Balato.

Guest Editor

Prof. Anna Balato, MD, PhD

Associate Professor of Dermatology, Dermatology Unit, University of Campania, Naples, Italy

ABSTRACT Atopic dermatitis (AD) is a prevalent inflammatory skin disorder affecting all age and ethnic groups. The age-dependent varying appearance and extent of pruritic lesions are accompanied by distinct individual suffering, highlighting the importance of effective treatment options. Over the past years systemic drugs have considerably extended therapeutic approaches of patients with moderate to severe $\mathrm{AD}$, in particular new biologics, most notably dupilumab has appeared as major breakthrough. In addition to monoclonal blockade of IL-4 and IL-13 pathway, more cytokines have been found to play a substantial role in $\mathrm{AD}$ pathogenesis, presenting potential targets for new therapy options. 


\section{Introduction}

Atopic dermatitis (AD), one of the most common inflammatory skin disorders affecting people of all ages and ethnicities, is characterized by typical age-dependent clinical features and substantial individual suffering as well as extensive economic impact $[1,2]$. Whereas infants often present acute eczematous pruritic lesions involving the face, cheeks, and trunk, children, adolescents, and adults develop rather diffuse lesions affecting the flexures, alternating between acute and chronic areas [3].

With numerous new emerging drugs expanding the clinical practice of $\mathrm{AD}$ healthcare in recent years, the approval of dupilumab, represented a major breakthrough in the therapy of patients with moderate to severe $\mathrm{AD}$. This review aims to describe currently available biological treatment options and potential future developments.

\section{Dupilumab}

The pathophysiology of $\mathrm{AD}$ is multifactorial, involving a genetic predisposition, epidermal barrier dysfunction, the skin microbiome, and type-2-T-helper-cells (Th2)-predominant inflammation [4-6]. The latter is mediated by various type-2 cytokines, among others interleukin-4 (IL-4), and IL-13. Moreover, IL-4 induces differentiation of Th-cells into Th2-cells, thus promoting further production of IL-4 and IL-13 [7]. Both using the same IL-4 receptor subunit alpha (IL-4R $\alpha$ ), these cytokines are responsible for IgE class switching in B-cells [8]. Given its key role in the pathway of type-2 mediated immune response, IL-4R $\alpha$ blockade was anticipated to represent a therapeutic approach to treating allergic diseases. Indeed, dupilumab, a human monoclonal antibody blocking IL-4R $\alpha$, was first shown to be effective in the treatment of $\mathrm{AD}$ in an early-phase randomized, double-blind, placebo-controlled trial in adults in 2014 [9]. Monotherapy of dupilumab at 4 weeks compared to placebo showed a rapid and dose-dependent 50\% improvement in the Eczema Area and Severity Index score (EASI-50) and decline in the pruritus numerical-rating scale. This effect was further intensified at 12 weeks [9]. Dupilumab was previously shown to be efficient in treating persistent, moderate-to-severe asthma with elevated eosinophil levels [10]. Simpson et al reported in 2016 an improvement of $75 \%$ in the EASI, a reduction of pruritus and improvement in quality of life in two phase- 3 trials of dupilumab versus placebo in $\mathrm{AD}$, comprising almost 1,400 patients aged 18 years and older in North America, Europe, and Asia.

Adverse effects were mild to moderate and comprised nasopharyngitis, upper respiratory tract infections, conjunctivitis, injection-site reactions, and exacerbation of $\mathrm{AD}$ [11]. In 2017, dupilumab was authorized for the treatment of AD in the European Union by the European Medicines Agency (EMA). Shortly after, phase-3 trials for adolescents [12] and children aged 6 years and older [13] were conducted, which showed an improvement in EASI, Investigator's Global Assessment (IGA) score, and quality of life compared to placebo with similar adverse effects in these age groups as well. Currently, the treatment of AD with dupilumab is authorized by EMA for patients aged 6 years and older. Recently, a promising phase-2, two-age cohort, two-dose level, multicenter study of dupilumab in the treatment of severe uncontrolled AD in children aged 6 months to $<6$ years has showed efficacy and a similar safety-profile as seen in older age groups [14]. These data could support a phase-3 trial of dupilumab in this patient population to further offer therapeutic approaches for topical corticosteroid-refractory $\mathrm{AD}$ in this age group.

\section{Further Biologics in Atopic Dermatitis' Treatment}

In addition to IL-4 and IL-13, other cytokines have been identified to play an important role in the pathophysiology of chronic inflammatory skin disease. These are IL-31, thymic stromal lymphopoietin (TSLP), IL-17, and IL-22 cytokines, which can also negatively affect the expression of barrier proteins.

\section{IL-13}

A Th-2 mediated exuberant immune response is stated to be the key mechanism in the pathogenesis of AD. Zheng et al induced pruritic dermatitis and skin remodeling in an IL-13 overexpressing mouse model. They showed that IL-13 was mainly produced in the skin and caused xerosis, itching lesions, chronic inflammation of the skin, and dermal infiltration of CD4+-cells, mast cells, and eosinophils. Mice models' skin also showed increased fibrosis and vascularization [15]. Another study was able to show a relative upregulation of IL-13 mRNA compared to IL-4 mRNA in lesional skin of AD patients, thus providing first evidence that IL-13 may be the crucial cytokine in the IL-4/IL-13 axis [16]. IL-13 as a potent stimulator of dermal inflammation and remodeling is another hypothesis for the pathogenesis of $\operatorname{AD}[17,18]$.

Both lebrikizumab and tralokinumab, monoclonal antibodies that target IL-13, have been recently investigated for AD treatment. The effectiveness of a therapy with Tralokinumab was analyzed in two 52-week, randomized, double-blind, multicenter, placebo-controlled phase-3-trials among more than 1,500 participants. Tralokinumab showed significant reduction in EASI and IGA scores in a dose-dependent manner compared to placebo. Participants receiving tralokinumab had a response rate of up to $70 \%$ for EASI-50 and up to $40 \%$ for EASI-75. In addition, early improvements were observed in pruritus, sleep disturbances, dermatology life quality index (DLQI), and the severity scoring of atopic dermatitis (SCORAD). Those effects were demonstrated in most participants at week 16 , and after extended use, at week 
52. The most common adverse reactions due to tralokinumab use were upper respiratory tract infections, conjunctivitis, and injection site reactions [19]. Based on these study results, the EMA recommended tralokinumab approval for the treatment of moderate to severe AD, in June 2021.

Like tralokinumab, lebrikizumab is an IL-13 antibody. Guttman-Yasky et al examined the effects of the biological lebrikizumab in a double-blind, placebo-controlled, 16-week, phase-2b study among 280 individuals who were randomly assigned to a placebo group or to groups receiving lebrikizumab in different doses. A rapid dose-dependent effect of lebrikizumab was described in clinical scores and symptoms when compared to placebo. For instance, after 16 weeks, an EASI improvement of up to $72.1 \%$ was shown in the group receiving $250 \mathrm{mg}$ lebrikizumab every 2 weeks compared to placebo $(\mathrm{P}<0.001)$. Adverse events were mainly injection site reactions, herpes virus infections, and conjunctivitis. No adverse events led to premature patient discontinuation. If the positive effect of this biological can be reproduced in currently underway phase-3 studies, lebrikizumab can be considered a highly effective therapy modality in the treatment of moderate to severe $\mathrm{AD}[20]$.

\section{IL-31}

Intense itching is one of the greatest burdens of $\mathrm{AD}$ patients. IL-31, a cytokine primarily produced by CD4+ T-cells, has been found to be increased in skin samples of $\mathrm{AD}$ patients compared to healthy subjects [21]. Takamori et al studied IL-31-deficient mice models. Interestingly these showed decreased scratch frequency and duration, during induced contact dermatitis [22]. Treatment with IL-31-antibodies also showed reduced scratching behavior in $\mathrm{AD}$-induced $\mathrm{NC} / \mathrm{nga}$ mice $[17,18,23,24]$.

In a randomized, double-blind, placebo-controlled study, Ruzicka et al investigated the safety and efficacy of nemolizumab, a humanized antihuman IL-31a receptor antibody, in combination with topical steroids in patients with $\mathrm{AD}$. The results showed that subcutaneous administration of Nemolizumab was well tolerated by all subjects. Nemolizumab significantly decreased pruritus compared to placebo $(\mathrm{P}<0.01)$, but no significant reduction in EASI was observed. Accordingly, nemolizumab appears to be another therapeutic option, especially for AD patients suffering from pruritus [24, 25]. Currently, there are 3 ongoing clinical phase- 3 studies to prove nemolizumab safety and efficacy among a larger number of patients suffering from AD (NCT03989349, NCT03989206, NCT03985943, clinicaltrials.gov).

\section{Thymic Stromal Lymphopoietin and OX40}

The TSLP is a cytokine that is upregulated by IL-13 and directly leads epidermic dendritic cells (DC) to a Th-2-response. TSLP is mainly produced in keratinocytes and appears to play a key role in the activation of DC. Due to allergen damage, keratinocytes express TSLP, which in turn activates DC, thus increasing their expression of OX40L. OX40L leads to the differentiation of naive $\mathrm{CD}^{+}{ }^{+}$-cells into inflammatory Th-2-memory cells. OX40L is expressed by DC and activates T-cells and memory cells. The interaction between OX40 and OX40L appears to be critical for the long-term survival of $\mathrm{CD} 4{ }^{+} \mathrm{T}$-cells, which are responsible for inflammation in $\mathrm{AD}[17,18]$.

There is evidence that mice overexpressing TSLP develop $\mathrm{AD}$ and that their lesional skin contains increased numbers of Th- 2 cells. Current studies are investigating whether this pathway can be used for new therapeutic options for the treatment of AD. Guttman-Yassky et al investigated the efficacy and safety of GBR830, a humanized antibody against OX40, in an explorative phase-2a, placebo-controlled study among patients with moderate to severe AD. First results showed that GBR380 was well tolerated and showed a significant reduction in Th1-, Th2, and Th17/22 expression in lesional skin compared to placebo $(\mathrm{P}<0.01)$. Furthermore, a significant reduction in the epidermal thickness could be observed $(\mathrm{P}<0.001)[26]$. The phase- $2 \mathrm{~b}$ trial is completed, but results have not yet been published (NCT03568162).

A phase-2a study from Japan, which also showed promising results, investigated KHK4083, a monoclonal antibody against OX40. A continuous reduction of EASI and IGA scores was achieved in this study. Furthermore, KHK4083 showed an acceptable safety profile [27].

Tepezelumab, a monoclonal antibody, is another medication targeting TSLP. In a phase-2 study, 113 patients were 1:1 randomized and treated either with placebo or subcutaneous Tepezelumab every 2 weeks. Results of this trial showed that a higher percentage of patients treated with Tepezelumab reached an EASI50 after 12 weeks compared to the placebo group; however, the effect was not significant $(P=0.91)$ [28]. To show possible beneficial effects of this treatment option, larger studies need to be conducted.

\section{$I L-33$}

IL-33 is another key cytokine involved in AD pathogenesis. Increased amounts of IL-33 have been detected in AD patients' lesional skin as well as in mice models. Furthermore, IL-33 has been shown to induce Th-2 immune response and significantly promote the release of IL-4, IL-5, and IL-13 as well as increase the activity of OX40L. Blocking IL-33 and subsequently suppressing the mentioned cytokines could also be leveraged in the therapy of $\mathrm{AD}$ [29-31]. The first effects of an IL-33 inhibition were showed in a mouse model [32].

In a phase-2a study, Chen et al examined the in-vivo effect of etokimab, (ANB020), a monoclonal IgG-antibody, in 12 patients with moderate to severe AD. After a single systemic administration of etokimab, $83 \%$ of patients achieved EASI50 and $33 \%$ EASI-75 at day 29. A significant reduction in 
Table 1. Overview of Biologicals Approved or Tested for Atopic Dermatitis Treatment.

\begin{tabular}{|l|l|l|l|}
\hline \multicolumn{1}{|c|}{ Biological } & \multicolumn{1}{|c|}{ Target } & Current phase of clinical trials & \multicolumn{1}{|c|}{ Main findings in clinical trials } \\
\hline Dupilumab & IL-4/IL-13 & $\begin{array}{l}\text { Approved for clinical use by } \\
\text { EMA }\end{array}$ & $\begin{array}{l}\text { EASI-75 improvement after 16-weeks of trial } \\
\text { (Phase III) [11] }\end{array}$ \\
\hline Tralokinumab & IL-13 & $\begin{array}{l}\text { Approved for clinical use by } \\
\text { EMA }\end{array}$ & $\begin{array}{l}\text { EASI-75 improvement \& IGA 0 or 1 after 16-weeks of } \\
\text { trial compared to placebo (Phase III) [19] }\end{array}$ \\
\hline Lebrikizumab & IL-13 & 3, still recruiting & $\begin{array}{l}\text { Up to 72.1\% improvement EASI (250mg dose) after } \\
\text { 16-weeks of trial compared to placebo (Phase IIb) [20] }\end{array}$ \\
\hline Nemolizumab & IL-31 & 3, still recruiting & $\begin{array}{l}\text { Up to 63.1\% change in the pruritus VAS score after } \\
\text { 12-weeks compared to placebo (Phase IIb) [24] }\end{array}$ \\
\hline GBR830/IBS830 & OX40 & $\begin{array}{l}\text { 2b, finished, results not } \\
\text { published }\end{array}$ & $\begin{array}{l}\text { EASI-50 improvement after 71 days compared to } \\
\text { placebo (Phase IIa) [26] }\end{array}$ \\
\hline KHK4083 & OX40 & $\begin{array}{l}\text { 2a, completed, results not } \\
\text { published }\end{array}$ & $\begin{array}{l}\text { Continued improvement in EASI and IGA } \\
\text { (Phase I) [27] }\end{array}$ \\
\hline Tepezelumab & TSLP & 2b, still recruiting & $\begin{array}{l}\text { Numerical EASI50 improvement after 12-weeks of } \\
\text { trial compared to placebo (Phase IIa) [28] }\end{array}$ \\
\hline Etokimab & IL-31 & 2b, still recruiting & $\begin{array}{l}\text { 83\% improvement of EASI50 and 33\% EASI75 after } \\
\text { 29-days of a single dose (Phase IIa) [33] }\end{array}$ \\
\hline Fezakinumab & IL-22 & 2a, completed & $\begin{array}{l}\text { SCORAD improvement greater compared to placebo } \\
\text { at 12-weeks of trial (Phase IIa) [36] }\end{array}$ \\
\hline
\end{tabular}

"Data from clinicaltrials.gov, Worm et al [18], \& Li et al [17].

neutrophile infiltration of the skin was also observed compared to placebo. These results suggested that IL-31 suppression can positively affect inflammatory responses and may represent another component of AD therapy [33]. A phase-2 trial has been initiated and is currently in the recruiting phase (NCT03533751).

\section{TH-22/IL-22}

$\mathrm{AD}$ is traditionally considered a Th-2-mediated disease. However, it has previously been shown that Th-22 cells also play an important role in pathogenesis. Th-22 cells express IL-22, which in turn activates a receptor responsible for epidermal hyperplasia, migration of keratinocytes, downregulation of keratinocytic differentiation, and elevation of proinflammatory cytokines. In vitro analyses of skin biopsies of patients showed a correlation between the severity of AD with the presence of CD8+-IL-22-cells [17, 34, 35].

Guttmann-Yasky et al performed a randomized, double-blind, placebo-controlled trial testing the efficacy and safety of fezakinumab, a monoclonal IgG-antibody against IL-22. A significant reduction in the SCORAD $(\geq 50)$ was found in the subgroup of patients with severe AD compared to placebo $(\mathrm{P}<0.029)$, but this effect was not found for the entire study population. At week 12, a significant reduction of the body surface area involvement was observed in all patients receiving fezakinumab compared to placebo. In addition, fezakinumab demonstrated a beneficial safety profile, with upper respiratory tract infections being the most reported adverse event, which will require further investigation in a larger study population [36].

\section{Conclusion}

New findings on the pathogenesis of $\mathrm{AD}$ introduce new therapeutic approaches almost daily as summarized in Table 1 and Figure 1. For example, the Th-17/IL-23 axis, which was long considered pathognomonic for psoriasis, could now also be proven to play a role in the pathogenesis of $\mathrm{AD}$ [37]. Already proven agents for the therapy of psoriasis are available and may be used for therapeutic approaches for patients suffering

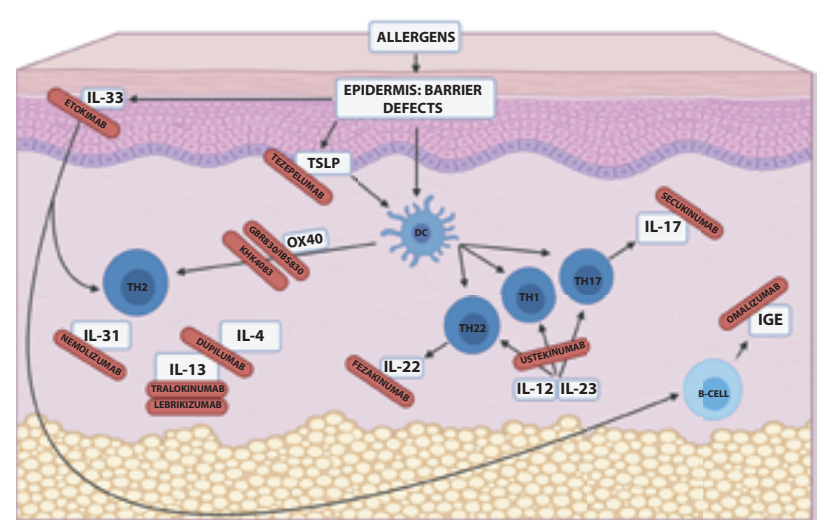

Figure 1. Pathogenesis and therapeutic targets in atopic dermatitis (AD). Modified according to Worm et al [35] and Li et al [14]. Increased skin penetration of allergens due to epidermal barrier defects results in type-2-T-helper-cells (Th2)-predominant inflammation with and without activation of dendritic cells (DC). Various cytokines contribute in the pathogenesis of $\mathrm{AD}$, representing effective and future possible therapeutic targets for biologics in combating AD. Cells that activate DC or those that are activated by them, such as TSLP or OX40, represent potential targets. 
from AD. Described findings and clinical results illustrate the viability of biologicals and support the endeavor of targeted, patient-specific medication for the treatment of $\mathrm{AD}$.

\section{References}

1. Ring J, Zink A, Arents BWM, et al. Atopic eczema: burden of disease and individual suffering - results from a large EU study in adults. J Eur Acad Dermatol Venereol. 2019;33(7):1331-1340. DOI: 10.1111/jdv.15634.PMID: 31002197.

2. Zink AGS, Arents B, Fink-Wagner A, Seitz IA, Mensing U, Wettemann N, de Carlo G, Ring J. Out-of-pocket Costs for Individuals with Atopic Eczema: A Cross-sectional Study in Nine European Countries. Acta Derm Venereol. 2019;99(3): 263-267. DOI: 10.2340/00015555-3102. PMID: 30521060.

3. Langan SM, Irvine AD, Weidinger S. Atopic dermatitis. Lancet. 2020; 396(10247):345-360. DOI: 10.1016/s01406736(20)31286-1.

4. Paternoster L, Standl M, Waage J, et al. Multi-ancestry genome-wide association study of 21,000 cases and 95,000 controls identifies new risk loci for atopic dermatitis. Nat Genet. 2015; 47(12): 1449-1456. DOI: 10.1038/ng.3424.

5. Jungersted JM, Scheer H, Mempel M, et al. Stratum corneum lipids, skin barrier function and filaggrin mutations in patients with atopic eczema. Allergy. 2010;65(7):911-8. DOI: 10.1111/j.13989995.2010.02326.x. PMID: 20132155.

6. Geoghegan JA, Irvine AD, Foster TJ. Staphylococcus aureus and Atopic Dermatitis: A Complex and Evolving Relationship. Trends Microbiol. 2018;26(6):484-497. DOI: 10.1016/j. tim.2017.11.008.PMID: 29233606.

7. Ho IC, Miaw SC. Regulation of IL-4 Expression in Immunity and Diseases. Adv Exp Med Biol.2016; 941: 31-77. DOI: 10.1007/978-94-024-0921-5_3. PMID: 27734408.

8. Gandhi NA, Bennett BL, Graham NMH, Pirozzi G, Stahl N, Yancopoulos GD. Targeting key proximal drivers of type 2 inflammation in disease. Nature Reviews Drug Discovery. 2016;15(1):3550. DOI: 10.1038/nrd4624.PMID: 26471366.

9. Beck LA, Thaçi D, Hamilton JD, et al. Dupilumab treatment in adults with moderate-to-severe atopic dermatitis. N Engl J Med. 2014; 371(2):130-9. DOI: 10.1056/NEJMoa1314768. PMID: 25006719.

10. Wenzel S, Ford L, Pearlman D, et al. Dupilumab in Persistent Asthma with Elevated Eosinophil Levels. New England Journal of Medicine. 2013;368(26): 2455-2466. DOI: 10.1056/NEJMoa1304048. PMID: 23688323.

11. Simpson EL, Bieber T, Guttman-Yassky E, et al. Two Phase 3 Trials of Dupilumab versus Placebo in Atopic Dermatitis. New England Journal of Medicine. 2016;375(24):2335-2348. DOI: 10.1056/ NEJMoa1610020. PMID: 27690741.

12. Simpson EL, Paller AS, Siegfried EC, et al. Efficacy and Safety of Dupilumab in Adolescents With Uncontrolled Moderate to Severe Atopic Dermatitis: A Phase 3 Randomized Clinical Trial. JAMA Dermatol. 2020;156(1): 44-56. DOI: 10.1001/jamadermatol.2019.3336. PMID: 31693077. PMCID: PMC6865265.

13. Paller AS, Siegfried EC, Thaçi D, et al. Efficacy and safety of dupilumab with concomitant topical corticosteroids in children 6 to 11 years old with severe atopic dermatitis: A randomized, double-blinded, placebo-controlled phase 3 trial. J Am Acad Dermatol. 2020;83(5):1282-1293. DOI: 10.1016/j.jaad.2020.06.054. PMID: 32574587.
14. Paller AS, Siegfried EC, Simpson EL, et al. A phase 2, open-label study of single-dose dupilumab in children aged 6 months to $<6$ years with severe uncontrolled atopic dermatitis: pharmacokinetics, safety and efficacy. J Eur Acad Dermatol Venereol. 2021;35(2):464-475. DOI: 10.1111/jdv.16928. PMID: 32893393. PMCID: PMC7894166.

15. Zheng T, Oh MH, Oh SY, Schroeder JT, Glick AB, Zhu Z. Transgenic expression of interleukin-13 in the skin induces a pruritic dermatitis and skin remodeling. J Invest Dermatol. 2009;129(3): 742-51. DOI: 10.1038/jid.2008.295. PMID: 18830273. PMCID: PMC4356214.

16. Tazawa T, Sugiura H, Sugiura Y, Uehara M. Relative importance of IL-4 and IL-13 in lesional skin of atopic dermatitis. Arch Dermatol Res. 2004;295(11): 459-64. DOI: 10.1007/s00403004-0455-6. PMID: 15014952.

17. Li R, Hadi S, Guttman-Yassky E. Current and emerging biologic and small molecule therapies for atopic dermatitis. Expert Opin Biol Ther. 2019;19(4):367-380. DOI: 10.1080/14712598.2019.1573422. PMID: 30672355.

18. Worm M, Francuzik W, Kraft M, Alexiou A. Modern therapies in atopic dermatitis: biologics and small molecule drugs. $J$ Dtsch Dermatol Ges. 2020;18(10): 1085-1092. DOI: 10.1111/ ddg. 14175 .

19. Wollenberg A, Blauvelt A, Guttman-Yassky E, et al. Tralokinumab for moderate-to-severe atopic dermatitis: results from two 52-week, randomized, double-blind, multicentre, placebo-controlled phase III trials (ECZTRA 1 and ECZTRA 2). Br J Dermatol. 2021; 184(3): 437-449. DOI: 10.1111/bjd.19574. PMID: 33000465 . PMCID: PMC7986411.

20. Guttman-Yassky E, Blauvelt A, Eichenfield LF, et al. Efficacy and Safety of Lebrikizumab, a High-Affinity Interleukin 13 Inhibitor, in Adults With Moderate to Severe Atopic Dermatitis: A Phase 2b Randomized Clinical Trial. JAMA Dermatol. 2020;156(4):411420. DOI: 10.1001/jamadermatol.2020.0079. PMID: 32101256. PMCID: PMC7142380.

21. Nobbe S, Dziunycz P, Mühleisen B, et al. IL-31 expression by inflammatory cells is preferentially elevated in atopic dermatitis. Acta Derm Venereol. 2012; 92(1):24-8. DOI: 10.2340/000155551191. PMID: 22041865.

22. Takamori A, Nambu A, Sato K, et al. IL-31 is crucial for induction of pruritus, but not inflammation, in contact hypersensitivity. Scientific Reports. 2018; 8(1):6639. DOI: 10.1038/s41598-01825094-4. PMID: 29703903. PMCID: PMC5923199.

23. Grimstad O, Sawanobori Y, Vestergaard C, Bilsborough J, Olsen UB, Grønhøj-Larsen C, Matsushima K. Anti-interleukin-31-antibodies ameliorate scratching behaviour in NC/Nga mice: a model of atopic dermatitis. Exp Dermatol.2009; 18(1):35-43. DOI: 10.1111/j.1600-0625.2008.00766.x. PMID: 19054054.

24. Ruzicka T, Hanifin JM, Furue M, et al. Anti-Interleukin-31 Receptor A Antibody for Atopic Dermatitis. N Engl J Med. 2017;376(9):826-835. DOI: 10.1056/NEJMoa1606490. PMID: 28249150.

25. Nemoto O, Furue M, Nakagawa H, et al. The first trial of CIM331, a humanized antihuman interleukin-31 receptor A antibody, in healthy volunteers and patients with atopic dermatitis to evaluate safety, tolerability and pharmacokinetics of a single dose in a randomized, double-blind, placebo-controlled study. $\mathrm{Br}$ J Dermatol. 2016;174(2): 296-304. DOI: 10.1111/bjd.14207. PMID: 26409172.

26. Guttman-Yassky E, Pavel AB, Zhou L, et al. GBR 830, an anti-OX40, improves skin gene signatures and clinical scores 
in patients with atopic dermatitis. J Allergy Clin Immunol. 2019;144(2):482-493.e7. DOI: 10.1016/j.jaci.2018.11.053. PMID: 30738171.

27. Nakagawa H, Iizuka H, Nemoto O, Shimabe M, Furukawa Y, Kikuta N, Ootaki K. Safety, tolerability and efficacy of repeated intravenous infusions of KHK4083, a fully human anti-OX40 monoclonal antibody, in Japanese patients with moderate to severe atopic dermatitis. J Dermatol Sci. 2020;99(2):82-89. DOI: 10.1016/j.jdermsci.2020.06.005. PMID: 32651105.

28. Simpson EL, Parnes JR, She D, Crouch S, Rees W, Mo M, van der Merwe R. Tezepelumab, an anti-thymic stromal lymphopoietin monoclonal antibody, in the treatment of moderate to severe atopic dermatitis: A randomized phase 2 a clinical trial. J Am Acad Dermatol. 2019; 80(4): 1013-1021. DOI: 10.1016/j. jaad.2018.11.059. PMID: 30550828 .

29. Schmitz J, Owyang A, Oldham E, et al. IL-33, an interleukin-1-like cytokine that signals via the IL-1 receptor-related protein ST2 and induces $\mathrm{T}$ helper type 2 -associated cytokines. Immunity. 2005;23(5):479-90. DOI: 10.1016/j.immuni.2005.09.015. PMID: 16286016.

30. Murakami-Satsutani N, Ito T, Nakanishi T, et al. IL-33 promotes the induction and maintenance of Th2 immune responses by enhancing the function of OX40 ligand. Allergol Int. 2014;63(3):443-55. DOI: 10.2332/allergolint.13-OA-0672.

31. Cherry WB, Yoon J, Bartemes KR, Iijima K, Kita H. A novel IL-1 family cytokine, IL-33, potently activates human eosinophils. $J$ Allergy Clin Immunol. 2008;121(6):1484-90. DOI: 10.1016/j. jaci.2008.04.005. PMID: 18539196. PMCID: PMC2821937.
32. Peng G, Mu Z, Cui L, Liu P, Wang Y, Wu W, Han X. Anti-IL-33 Antibody Has a Therapeutic Effect in an Atopic Dermatitis Murine Model Induced by 2, 4-Dinitrochlorobenzene. Inflammation. 2018;41(1):154-163. DOI: 10.1007/s10753-017-0673-7. PMID: 28952069.

33. Chen YL, Gutowska-Owsiak D, Hardman CS, et al. Proof-ofconcept clinical trial of etokimab shows a key role for IL-33 in atopic dermatitis pathogenesis. Sci Transl Med. 2019;11(515). DOI: 10.1126/scitranslmed.aax2945. PMID: 31645451.

34. Nograles KE, Zaba LC, Shemer A, et al. IL-22-producing "T22" $\mathrm{T}$ cells account for upregulated IL-22 in atopic dermatitis despite reduced IL-17-producing TH17 T cells. J Allergy Clin Immunol. 2009;123(6): 1244-52.e2.DOI: 10.1016/j.jaci.2009.03.041. PMID: 19439349. PMCID: PMC2874584.

35. Noda S, Krueger JG, Guttman-Yassky E. The translational revolution and use of biologics in patients with inflammatory skin diseases. J Allergy Clin Immunol. 2015; 135(2):324-36.DOI: 10.1016/j.jaci.2014.11.015. PMID: 25541257.

36. Guttman-Yassky E, Brunner PM, Neumann AU, et al. Efficacy and safety of fezakinumab (an IL-22 monoclonal antibody) in adults with moderate-to-severe atopic dermatitis inadequately controlled by conventional treatments: A randomized, double-blind, phase 2a trial. J Am Acad Dermatol.2018;(78) 5: 872-881.e6. DOI: 10.1016/j.jaad.2018.01.016. PMID: 29353025.

37. Koga C, Kabashima K, Shiraishi N, Kobayashi M, Tokura Y. Possible pathogenic role of Th17 cells for atopic dermatitis. J Invest Dermatol. 2008;128(11):2625-2630. DOI: 10.1038/ jid.2008.111. PMID: 18432274. 